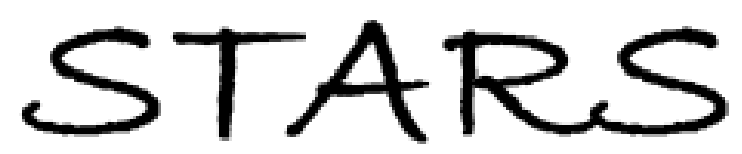

University of Central Florida

STARS

Faculty Bibliography 2010s

Faculty Bibliography

$1-1-2011$

\title{
Vertical field switching for blue-phase liquid crystal devices
}

\author{
Hui-Chuan Cheng \\ University of Central Florida \\ Jin Yan \\ University of Central Florida \\ Takahiro Ishinabe \\ University of Central Florida \\ Shin-Tson Wu \\ University of Central Florida
}

Find similar works at: https://stars.library.ucf.edu/facultybib2010 University of Central Florida Libraries http://library.ucf.edu

This Article is brought to you for free and open access by the Faculty Bibliography at STARS. It has been accepted for inclusion in Faculty Bibliography 2010 s by an authorized administrator of STARS. For more information, please contactSTARS@ucf.edu.

\section{Recommended Citation}

Cheng, Hui-Chuan; Yan, Jin; Ishinabe, Takahiro; and Wu, Shin-Tson, "Vertical field switching for blue-phase liquid crystal devices" (2011). Faculty Bibliography 2010s. 1171.

https://stars.library.ucf.edu/facultybib2010/1171

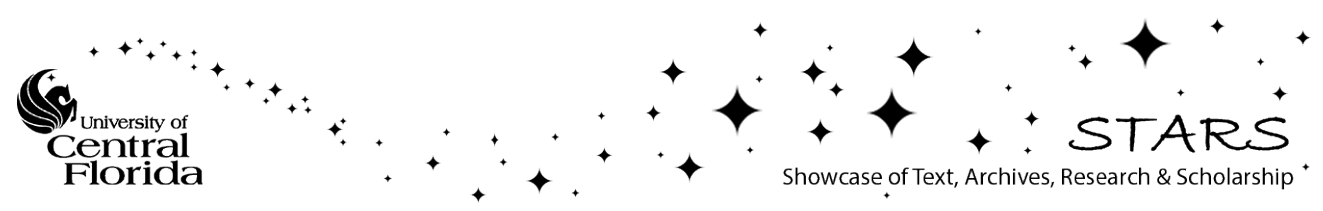




\section{Vertical field switching for blue-phase liquid crystal devices}

Cite as: Appl. Phys. Lett. 98, 261102 (2011); https://doi.org/10.1063/1.3604011

Submitted: 04 May 2011 . Accepted: 03 June 2011 . Published Online: 27 June 2011

Hui-Chuan Cheng, Jin Yan, Takahiro Ishinabe, and Shin-Tson Wu

\section{ARTICLES YOU MAY BE INTERESTED IN}

Low voltage blue-phase liquid crystal displays

Applied Physics Letters 95, 231101 (2009); https://doi.org/10.1063/1.3271771

Extended Kerr effect of polymer-stabilized blue-phase liquid crystals

Applied Physics Letters 96, 071105 (2010); https://doi.org/10.1063/1.3318288

Electro-optics of polymer-stabilized blue phase liquid crystal displays

Applied Physics Letters 94, 101104 (2009); https://doi.org/10.1063/1.3097355

\section{Applied Physics Reviews} Now accepting original research 


\title{
Vertical field switching for blue-phase liquid crystal devices
}

\author{
Hui-Chuan Cheng, Jin Yan, Takahiro Ishinabe, and Shin-Tson Wu ${ }^{\text {a) }}$ \\ College of Optics and Photonics, University of Central Florida, Orlando, Florida 32816, USA
}

(Received 4 May 2011; accepted 3 June 2011; published online 27 June 2011)

\begin{abstract}
A low-voltage $(<10 \mathrm{~V})$, high-transmittance $(>80 \%)$, submillisecond-response, and hysteresis-free polymer-stabilized blue-phase liquid crystal (BPLC) device with vertical field switching (VFS) and oblique incident light are demonstrated experimentally. Unlike the commonly employed in-plane switching in which the electric field is primarily in lateral direction and not uniform spatially, the VFS mode has uniform longitudinal field. As a result, the operating voltage is reduced by $\sim 3.2 \times$ which in turn helps to eliminate hysteresis. The VFS mode is a promising candidate for the emerging BPLC display and photonic devices. (C) 2011 American Institute of Physics. [doi:10.1063/1.3604011]
\end{abstract}

Polymer-stabilized blue-phase liquid crystal (PS-BPLC) ${ }^{1,2}$ is emerging as next-generation display and photonic technology because it exhibits some revolutionary features, e.g., no need for alignment layer, fast response time, and isotropic dark state. So far, the majority of previously demonstrated bluephase liquid crystal (BPLC) devices utilize in-plane switching (IPS) electrodes ${ }^{3-6}$ in which the electric field is mainly in lateral direction and the Kerr effect-induced birefringence is along the electric field if the employed BPLC has a positive dielectric anisotropy $(\Delta \varepsilon>0) .^{7}$ Therefore, the backlight can be easily set at normal incidence. However, IPS mode exhibits two major drawbacks: high operating voltage and relatively large hysteresis. The former is because the electric fields are confined near the electrodes and do not penetrate deeply into the BPLC bulk. ${ }^{8}$ Moreover, in an IPS mode, the electric fields are nonuniform spatially. Near electrode edges, the electric fields are particularly strong which could cause lattice deformation, known as electrostriction effect, leading to hysteresis. ${ }^{9,10}$ There is an urgent need to lower the operating voltage to $<10 \mathrm{~V}$ while completely eliminating the hysteresis.

In this letter, we propose a vertical field switching (VFS) BPLC device. Different from IPS mode, the electric field in the VFS cell is in longitudinal direction and is uniform. By using a thin cell gap and a large oblique incident angle $\left(70^{\circ}\right)$, the operating voltage is reduced to $\sim 10 \mathrm{~V}$ while eliminating the hysteresis. The uniform electric field also helps to reduce response time.

Figure 1 depicts the proposed VFS BPLC device structure. Because the electric field is in the longitudinal direction, only the incident light at an oblique angle $\left(0<\theta<90^{\circ}\right)$ can experience the phase retardation effect. For a given BPLC layer thickness, a larger $\theta$ results in a larger phase retardation, which helps to lower the operating voltage. Therefore, we need to couple the backlight to a large output angle (e.g., $\theta \sim 70^{\circ}$ ) and keep it reasonably well collimated. The reason for having a well-collimated light is to achieve better viewing symmetry. If the incident light is not well collimated, then the portion with a smaller angle would accumulate less phase retardation and form lower gray levels, while the larger angle part would accumulate more phase retardation and form higher gray levels. After the light exits to the viewer's side, the divergent backlight would cause asymmet-

a)Electronic mail: swu@mail.ucf.edu. ric brightness distribution when viewed from left side or right side of the panel. As a result, it will be difficult to obtain uniform brightness. In the following simulations, we choose the backlight with an angular distribution of $\sim 30^{\circ}$ full width at half maximum (FWHM).

The bottom coupling film laminated on the bottom polarizer that can substantially couple the oblique incident light to the bottom substrate and the BPLC layer. It has a prismatic structure which not only couples the oblique input light to the cell but also keeps a large angle. If we do not use the bottom coupling film, the refracted angle in the BPLC layer will be reduced dramatically by Snell's law. As a result, the phase retardation will be smaller and the operating voltage higher. The purpose of top coupling film on the top substrate is to couple the light to the air. Without the top coupling film, the oblique angle light will be trapped in the cell module because of total internal reflection (TIR). From our simulation results, we found that the prism structures of the coupling films with internal angles of $70^{\circ}-20^{\circ}-90^{\circ}$ can substantially couple the $\sim 70^{\circ}$ incident light to the substrate without changing the light direction and efficiently couple the light out of the substrate without TIR. The prism pitch should be smaller than each pixel size. Typically, it is from $\sim 5 \mu \mathrm{m}$ to $\sim 100 \mu \mathrm{m}$. Placed on the top coupling film, the turning film can steer the direction of the oblique light to the viewer's position by TIR. We design the irregular prism structure of turning film to spread the light to a wider range for larger viewing angle and more uniform brightness.

To confirm the advantages of our VFS device over the conventional IPS structure, we performed some experiments. Figure 2 shows the experimental setup for our VFS cell. To simulate the beam propagation path shown in Fig. 1, we prepared a transparent container filled with Glycerol $(n=1.47$ at $\lambda=633 \mathrm{~nm}$ ). The cell was immersed in the Glycerol liquid and it can be oriented at an arbitrary angle. Because of the index matching of glass and Glycerol, the light can pass through the BPLC in a very large angle. We prepared a polymerstabilized BPLC material using Chisso JC-BP01M. The liquid crystal (LC) host has a birefringence $\Delta \mathrm{n} \sim 0.17$ and $\Delta \varepsilon \sim 94$. After UV stabilization, the blue-phase temperature range is from $<0{ }^{\circ} \mathrm{C}$ to $\sim 70{ }^{\circ} \mathrm{C}$ and its Kerr constant is $K \sim 13 \mathrm{~nm} / \mathrm{V}^{2}$ at $23{ }^{\circ} \mathrm{C}$ and $\lambda=633 \mathrm{~nm} .{ }^{11}$ To make a fair comparison, we used the same material for both IPS and VFS cells. The IPS cell has patterned ITO electrodes with 


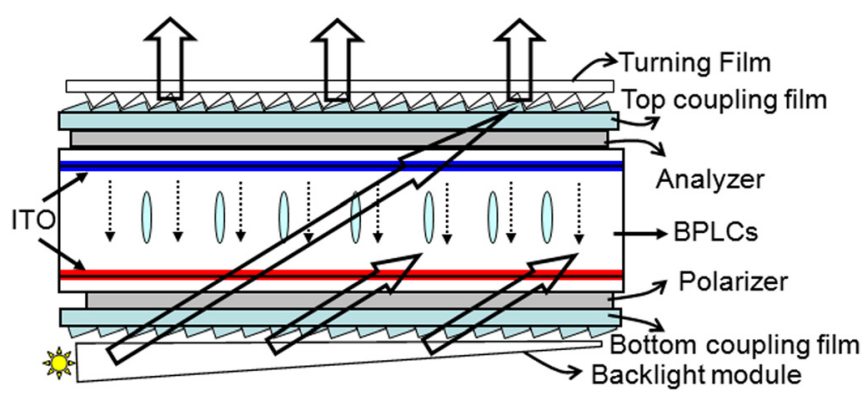

FIG. 1. (Color online) Device structure of the proposed VFS-BPLCD.

$10-\mu \mathrm{m}$ electrode width and $10-\mu \mathrm{m}$ electrode gap, and the cell gap is $d \sim 7.5 \mu \mathrm{m}$. In the VFS cells, both top and bottom glass substrates were over-coated with a thin $(\sim 80 \mathrm{~nm})$ ITO electrode; no polyimide layer was used. The cell gap was controlled at $d \sim 5.74 \mu \mathrm{m}$.

Figure 3 depicts the measured voltage-dependent transmittance (VT) curves (at $\lambda=633 \mathrm{~nm}$ and $\mathrm{T} \sim 23{ }^{\circ} \mathrm{C}$ ) of the IPS cell (at normal incidence) and VFS cell at $\theta=70^{\circ}$. For the IPS cell, the peak voltage occurs at $\mathrm{V}_{\mathrm{p}} \sim 50 \mathrm{~V}_{\mathrm{rms}}$. For the VFS cell at $\theta=70^{\circ}$, its $\mathrm{V}_{\mathrm{p}} \sim 16 \mathrm{~V}_{\text {rms }}$ which is $\sim 3.2 \times$ lower than that of IPS structure. To further reduce operating voltage, we could use a larger Kerr constant BPLC or increase the incident angle.

Hysteresis is a common phenomenon for polymer-stabilized liquid crystal devices. For display applications, hysteresis affects the accuracy of gray scale control and should be eliminated. Hysteresis is defined by the voltage difference $(\Delta \mathrm{V})$ at half-maximum transmittance when we scan the voltage forward and backward. From Fig. 3, the measured $\Delta \mathrm{V} /$ $\mathrm{V}_{\mathrm{p}}$ is $\sim 5.8 \%$ for the IPS cell. For our VFS cell with $\mathrm{d}=5.74$ $\mu \mathrm{m}$ and $\theta=70^{\circ}$, it is free from hysteresis! The reason is because our VFS cell has a lower operating voltage so that the electrostriction effect does not occur. On the contrary in an IPS cell, the generated electric fields are not uniform spatially. The electric fields are much stronger near the pixel edges than those in the electrode gap. The strong electric fields could cause lattice deformation locally which in turn contributes to hysteresis.

We also compared the response time of our VFS cell and IPS cell at $23^{\circ} \mathrm{C}$. The applied voltage swings from 0 to $16 \mathrm{~V}$ for the VFS cell and 0 to $50 \mathrm{~V}$ for the IPS cell. The [rise time, decay time] for the IPS cell and VFS cell are [648 $\mu \mathrm{s}, 1795 \mu \mathrm{s}]$, and [730 $\mu \mathrm{s}, 870 \mu \mathrm{s}]$, respectively. Our VFS mode shows a $\sim 2 \times$ faster decay time than IPS. Again, this is attributed to the smaller LC reorientation angle and uniform field of the VFS cell.

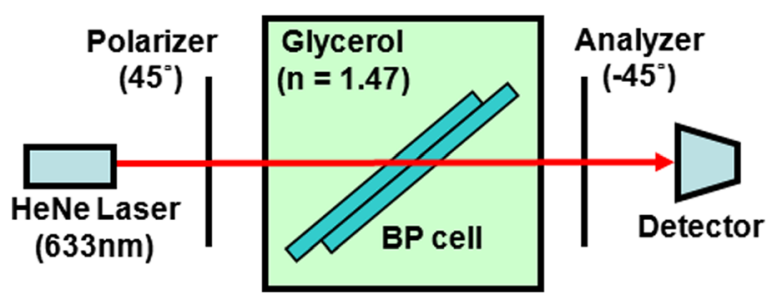

FIG. 2. (Color online) Experimental setup for characterizing the VFS cell.

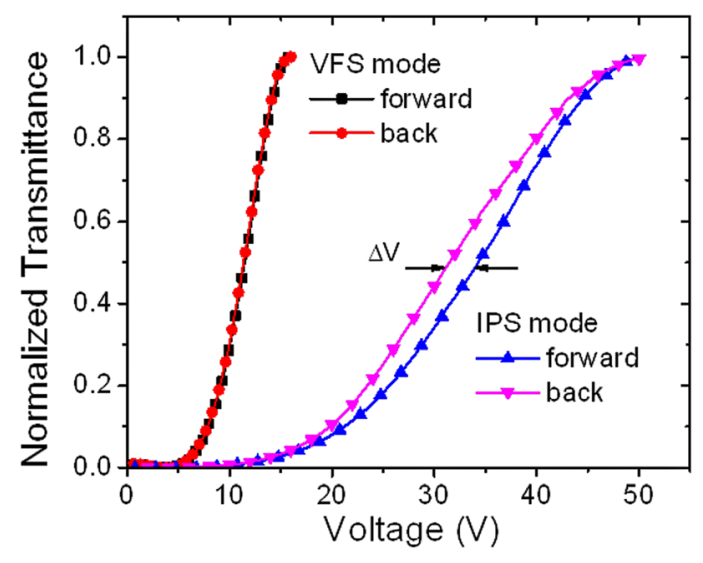

FIG. 3. (Color online) Measured VT curves and hysteresis of IPS and VFS cells. $\lambda=633 \mathrm{~nm}$.

Unlike IPS cell whose VT curve is insensitive to the cell gap, the peak transmittance voltage of our VFS cell is dependent on the cell gap. Two factors have to be considered: induced birefringence and effective cell gap. For a given voltage $\mathrm{V}$, as the cell gap decreases the electric field gets stronger because $\mathrm{E}=\mathrm{V} / \mathrm{d}$. According to Kerr effect, the induced birefringence is proportional to $E^{2}$. Thus, the induced birefringence is larger as the cell gap decreases. On the other hand, for a given incident angle, the beam path in the BPLC cell for accumulating phase retardation decreases as the cell gap decreases. Therefore, there ought to be an optimal cell gap for achieving certain phase retardation. If the cell gap is too thin, although the induced birefringence is large, the optical beam path is decreased so that the accumulated phase may not be adequate for achieving maximum transmittance. Moreover, the saturation phenomenon in the high field region should be also considered. In real situation, the induced birefringence will gradually saturate as the electric field increases. Therefore, the driving voltage could not decrease indefinitely when we use an ultra-thin cell gap, as described by the extended Kerr effect equation. ${ }^{12}$ In the extended Kerr effect model, the induced birefringence is expressed as $\Delta \mathrm{n}=\Delta \mathrm{n}_{\text {sat }}\left(1-\exp \left[-\left(\frac{E}{E_{S}}\right)^{2}\right]\right)$, where $\Delta \mathrm{n}_{\text {sat }}$ stands for the saturated induced birefringence and $E_{S}$

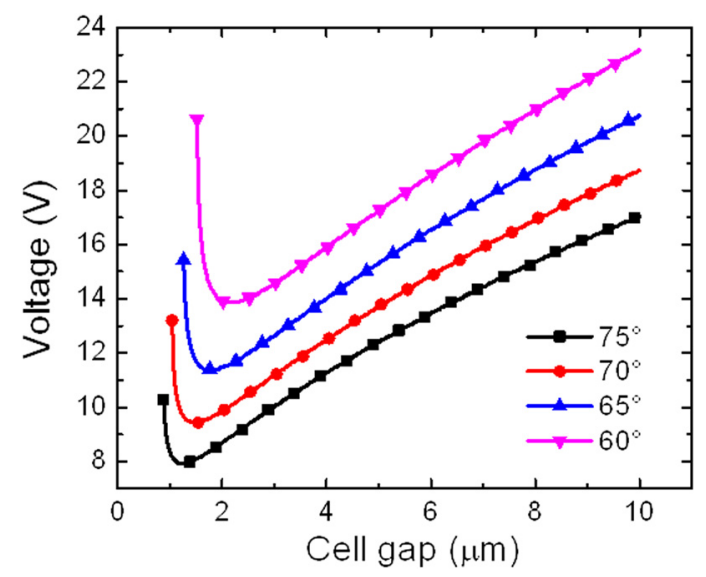

FIG. 4. (Color online) Simulated operating voltage vs. cell gap at different incident angles of VFS cells. $\lambda=550 \mathrm{~nm}$ and Kerr constant is assumed to be $K \sim 7.5 \mathrm{~nm} / \mathrm{V}^{2}$. 


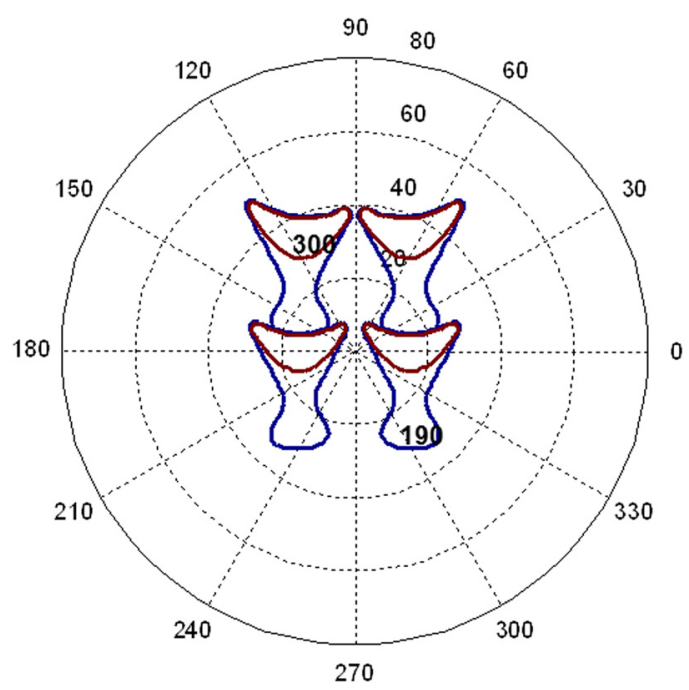

FIG. 5. (Color online) Isocontrast plot of VFS cell using irregular prism structure of turning film. Backlight FWHM $=30^{\circ}, \theta=70^{\circ}$, and $\lambda=550 \mathrm{~nm}$.

represents the saturation field. From the measured results in Fig. 3, our polymer-stabilized BPLC material has $K \sim 7.5$ $\mathrm{nm} / \mathrm{V}^{2}$ at $\lambda=633 \mathrm{~nm}$ in the low field region, with $\Delta n_{\text {sat }} \sim 0.17$ and $E_{s} \sim 6 \mathrm{~V} / \mu \mathrm{m}$. Based on the extended Kerr effect model, we simulated the cell gap versus the driving voltage at $\lambda=550 \mathrm{~nm}$ as shown in Fig. 4 . When the cell gap decreases, the driving voltage decreases and then reaches a minimum. For the case of $\theta=70^{\circ}$, the minimum peak voltage is $\mathrm{V}_{\mathrm{p}} \sim 9.5 \mathrm{~V}$ with $\mathrm{d} \sim 1.5 \mu \mathrm{m}$. We can optimize cell gap according to the material properties and incident angle to obtain the minimum voltage.

Typically, the optical efficiency of the IPS BPLC cell is $\sim 70 \%$ due to some dead zones above the electrodes. Our VFS BPLC cell can achieve $100 \%$ transmittance because of its uniform vertical fields without dead zones. The major optical loss comes from Fresnel reflection of the coupling films and turning films. From our simulations, the optical efficiency for the added films is over $85 \%$. Therefore, VFS should have comparable or higher optical efficiency than IPS.

Achieving wide viewing angle is a major challenge for the VFS mode because the backlight's incident angle is fairly large. In our simulation, we assume the absorption axes of the polarizer and analyzer (SEG1425DU, Nitto Denko) are at $45^{\circ}$ and $-45^{\circ}$, respectively, and two biaxial films are set at $48.8^{\circ}$ and $-48.5^{\circ}$. The $\mathrm{N}_{\mathrm{z}}$ factor, which is defined as $\left(\mathrm{n}_{\mathrm{z}}-\mathrm{z}_{\mathrm{y}}\right) /\left(\mathrm{n}_{\mathrm{x}}-\mathrm{n}_{\mathrm{y}}\right)$, is 0.4 for both biaxial films and $d\left(n_{x}-n_{y}\right)$ $=250 \mathrm{~nm}$, here $d$ is the thickness of biaxial films. The contrast ratio over 100:1 is limited to $\sim 20^{\circ}$ viewing cone if we use a regular prism-based turning film to steer the light to the viewer. This is because the backlight we used has a fairly narrow FWHM. Therefore, this design is suitable for narrow-view applications. To widen the viewing angle, we can design irregular prism structure of turning film to orient backlight to multiple directions. Figure 5 shows an example that the prisms of turning film are with two different angles. The high contrast contour extends to more locations. To spread the light more uniformly, a diffuser could be considered. We can also design more prism angles or use roundshape prisms to spread the light by TIR to get uniform brightness and high contrast ratio. The shape and angle of the micro prisms should be optimized according to the backlight profile employed.

In conclusion, the VFS-BPLC offers superior performances to IPS in lower operating voltage, higher transmittance, free from hysteresis, and faster response time. However, it is more sensitive to the cell gap than IPS. This operation mode can be applied to different display and photonic devices that require fast response time, such as field sequential color displays using RGB LED backlight.

The authors are indebted to AU Optronics for financial support.

${ }^{1}$ H. Kikuchi, M. Yokota, Y. Hisakado, H. Yang, and T. Kajiyama, Nature Mater. 1, 64 (2002).

${ }^{2}$ Y. Haseba, H. Kikuchi, T. Nagamura, and T. Kajiyama, Adv. Mater. 17, 2311 (2005).

${ }^{3}$ Z. Ge, S. Gauza, M. Jiao, H. Xianyu, and S. T. Wu, Appl. Phys. Lett. 94, 101104 (2009).

${ }^{4}$ Z. Ge, L. Rao, S. Gauza, and S. T. Wu, J. Disp. Technol. 5, 250 (2009).

${ }^{5}$ M. Kim, M. S. Kim, B. G. Kang, M. K. Kim, S. Yoon, S. H. Lee, Z. Ge, L. Rao, S. Gauza, and S. T. Wu, J. Phys. D 42, 235502 (2009).

${ }^{6}$ M. Jiao, Y. Li, and S. T. Wu, Appl. Phys. Lett. 96, 011102 (2010).

${ }^{7}$ L. Rao, Z. Ge, and S. T. Wu, Opt. Express 18, 3143 (2010).

${ }^{8}$ L. Rao, Z. Ge, S. T. Wu, and S. H. Lee, Appl. Phys. Lett. 95, 231101 (2009).

${ }^{9}$ M. Sato and A. Yoshizawa, Adv. Mater. 19, 4145 (2007).

${ }^{10}$ K. M. Chen, S. Gauza, H. Xianyu, and S. T. Wu, J. Disp. Technol. 6, 318 (2010).

${ }^{11}$ L. Rao, J. Yan, S. T. Wu, S. Yamamoto, and Y. Haseba, Appl. Phys. Lett. 98, 081109 (2011).

${ }^{12}$ J. Yan, H. C. Cheng, S. Gauza, Y. Li, M. Jiao, L. Rao, and S. T. Wu, Appl. Phys. Lett. 96, 071105 (2010). 